\title{
Data-collection strategies in SKED
}

\author{
BRUCE E. HAMILTON \\ The American University, Washington, D.C. 20016
}

\begin{abstract}
One advantage of the use of computers in the behavioral sciences is in the area of data collection. Regardless of the specialty area, the more sophisticated the issue being addressed, typically the greater the need for a more detailed analysis of data. This paper traces data-recording strategies in SKED from the simple counting of events to advanced-time and correlational analyses. Examples of common measures, such as interresponse times, and advanced measures, like sequential dependencies, will be discussed.
\end{abstract}

The goal of this paper is to acquaint the reader with a variety of data-collection strategies that have been written for the SKED notational language, and that have been implemented successfully in several laboratories. The strategies presented can be subdivided into three sections based upon their relative complexity. The first subdivision concerns the counting of discrete events and is the simplest strategy to use. This section records the usual data found with standard relay-rack programming. The second subdivision presents strategies which have been implemented in relay-rack and solid-state programming logic, but have been of limited use due to the expense of the large number of counters and other programming equipment necessary to implement them. An example of this type would be the classic interresponse-time (IRT) distribution. Data-collection routines of this sort require the use of the $F 1$ and F2 functions of SKED. Therefore, this section will be prefaced with a discussion of these functions sufficient to acquaint the reader with the basics of this mode of programming.

The third section will deal with strategies which are the most complex and, sometimes, the most easily programmed. These strategies use the F3 function. A discussion of $\mathrm{F} 3 \mathrm{~s}$ and their use are presented in this section, but the reader is advised to read Snapper and Hamilton (1974) for a more detailed account of F3s. A discussion of some basic programming considerations in data-collection problems will follow, with the goal being the transformation of the author's experiences into a few generalized rules to guide new programmers.

\section{SECTION 1: TRADITIONAL MEASUREMENTS}

The most frequently used method of assessing behavior in the behavioral sciences is the totaling of

This research was supported by NIMH Grant 1 R01 MH2281-03, Alan Silberberg, principal investigator, and by a Research Scientist Development Award K2-MH-70483 to Arthur G. Snapper from NIMH. The author wishes to thank Drs. Silberberg and Snapper for their comments and help. Reprints may be obtained from Bruce Hamilton, 321 Asbury Building, The American University, Washington, D.C. 20016. responses. This is followed in frequency of use by the recording of the duration in time of some event, such as stimulus conditions, latencies, or the like. SKED has these capabilities and is easily used to record these measures. A brief review of the structure of SKED is presented below to facilitate the discussion of these measures.

SKED is composed of a series of transition statements arranged in state sets. Each transition statement (see Snapper \& Kadden, 1970) is composed of three basic elements. The first element is the input criterion which determines when this transition statement is to take effect. This criterion can take one of three forms: responses ( $\mathrm{R})$, time ("), or Z-pulses (Z). Responses are numbered and correspond in a 1:1 manner with behavior. Time is calibrated from milliseconds to minutes. Z-pulses have no real-world counterpart and are thought of as timing pulses generated by the second element of other transition statements.

The second element of the transition statement is the output section. This section contains the list of events which occur contingent upon the input criterion. This section may contain stimulus-on or off statements, Z-pulses, but, of more interest, it may increment counters. The notation for incrementing is of the form $\mathrm{CN}$ where $\mathrm{N}$ is the counter number.

The final section of a transition statement is the next-state designation, $\mathrm{SN}$, where $\mathrm{N}$ is the number of some state in the present state set.

This discussion now brings us to the point of being able to specify the most basic types of recording schemes, the counting of discrete events. By including a counter reference in the output section or a transition statement, the frequency with which a transition occurs can be counted. The following state table would record every occurrence of an R1 in Counter 1 during the execution of this program.

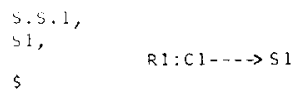

By imposing additional criteria on the state table, we may count only those transitions contingent upon $5 \mathrm{R} 1 \mathrm{~s}$ 
which occur between occurrences of a second response (R2). Remember that each entry into a state resets all counters which keep track of the number of responses, Z-pulses, or amount of time necessary for each transition.

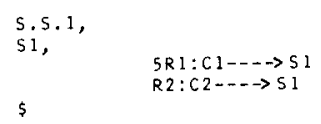

In a similar manner, counters may be used to simulate running-time meters. The asterisk after the counter number indicates that more counts are expected than the counter can hold so the next counter is reserved to count the number of times that Counter 1 overflows. The next counter is thereby used and must not be referenced. However, it must be included in the total number of counters reported to the compiler.

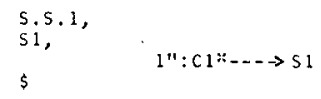

Using this type of format, we can now record the duration of a response if we have $R 1$ represent the closure (normally open) of a microswitch and R2 represent the release (normally closed) of the same switch.

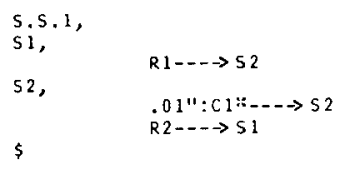

We may also record the latency to a response in the following manner:

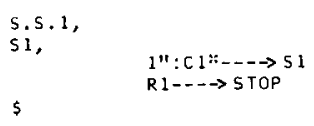

We may also record the occurrence of Z-pulses, as they are legitimate input criteria. In this example, we could have placed the counter directly into the time expression in S.S.1, but it is sometimes convenient to program this way.

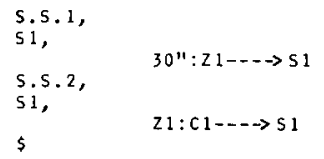

A more advanced recording scheme is accomplished by using the tag function of SKED and making the counting of a response contingent upon the tag value. For instance, suppose we have a multiple schedule and want to keep responses separate for the two components, with State Set 1 defined as $\mathbf{A}$.

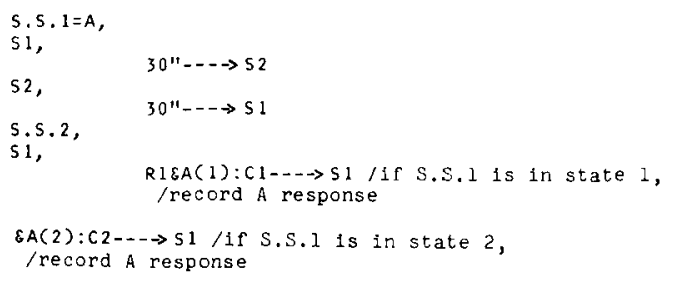

This use of tags and the gating function opens up new possibilities in recording events which amount to an initial sorting of data on-line. This brings us to the next section.

\section{SECTION 2: ON-LINE SORTING OF DATA}

The previous section contained a reference to the use of tags to help in the identification of a response-that is, was the response made in the first or second component of the multiple schedule? Tags can be used much more extensively, and such on-line sorting procedures as interresponse-time distributions (IRTs) can be generated. The reader must have an idea of what tags are and how they work before this section will be clear. A short review is presented here, but it is by no means complete and the reader is advised to read other accounts (cf. Snapper \& Kadden, 1970) for more details.

SKED contains certain key words, such as R, Z, ", $\mathrm{ON}, \mathrm{OFF}$, etc., which constitute the basic processes to be executed or the transition criteria. All of these key words require additional arguments, in the form of integer numbers, to specify completely which one and how many are to occur. A list of examples follow:

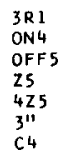

SKED has the ability to allow a variable to take the place of these integer numbers in the same way that $X$ stands for an unknown number in an algebraic expression. This variable is called a tag. It can take the place of integer numbers in transition statements and has the advantage of being manipulatable by the state table and by the experimenter via the Teletype. If the previous list of examples was rewritten using tags, they might appear as follows:

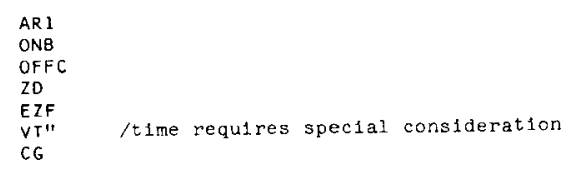

Several restrictions apply to the use of tags, namely: a tag must be defined only once in a state table; is always zero initially; and when used with outputs, care must be 
taken to insure that the proper octal number is used. To get around the problem of defining a tag only once, instead of each input event incrementing the tagged counter, it outputs a Z-pulse which, in turn, increments the counter. Thus, any time you wish to increment a tagged counter, you output the appropriate Z-pulse. As stated above, tags are manipulatable by the state table under program control. The notational devices used are the $F$ functions, specifically $F 1$ and $F 2$. For convenience, let us consider F2s first. F2s take the form of F2 (tag, \pm value), where tag equals a letter of the alphabet (except the letter $P$ ), and value equals a position or negative number between -4096 and +4096. F2s are used to set tags initially to a value and to set to other values.

$\mathrm{F} 1 \mathrm{~s}$, on the other hand, are used to change the value of the tag by known values until a limit is reached. The format of an Fl is Fl(tag, \pm incr, \pm limit) where tag is again a letter of the alphabet, incr is a position or negative number indicating by how much and in which direction (positive or negative) the tag value is to change, and limit is the positive or negative number designating the maximum (or minimum) value the tag may have. For example, consider the following state set:

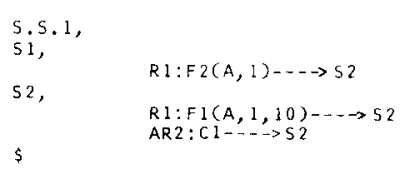

In this example, an $\mathrm{R} 1$ in $\mathrm{S} 1$ will set $\mathrm{A}$ to one and transfer control to $S 2$. In $S 2$, each $R 1$ increments the value of $A$ until the limit (10) is reached. Tag A cannot exceed 10 and must have a value following the rule $1 \leqslant A \leqslant 10$. $A$ is defined in $S 2$ as the number of occurrences of R2 which must occur before Counter 1 is incremented. Thus, the criterion number for the $\mathrm{R} 2$ transition statement depends on the value of $A$. R1 could itself be tagged, giving rise to the expression $\mathrm{RB}: \mathrm{F} 1(\mathrm{~A}, 1,10)-\cdots \mathrm{S} 2$. In this expression, which response is to be the criterion response depends upon the value of $\mathrm{B}$. In a similar manner, Counter 1 (C1) in the example could be tagged giving the counter expression CD. Which counter is to be incremented would then depend on the value of $\mathrm{D}$. It is this last instance (the tagging of a counter number) in which we are most interested. This leads us to the main discussion of the on-line sorting of data.

One of the simplest uses of the F functions results in a distribution of counters which represents total responses per reinforcement. For example:

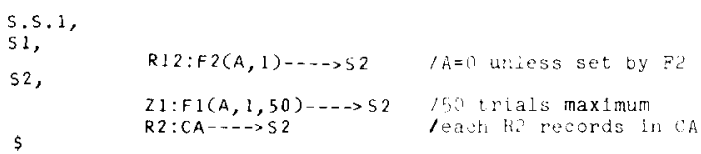

where $\mathrm{Z} 1$ is generated by the occurrence of refinforcement. All responses would go into Counter 1 until the first reinforcement, at which time Tag A is set to two. Responses then go into Counter 2 and so on. By changing the sriterion input $(\mathrm{Z} 1)$ in the above example to a time statement, we achieve a distribution of responses in time. Usually, this would be written so that reinforcement (Z1) would use the F2 function to reset Tag A to one, so that the distribution starts with reinforcement (reinforcement is time zero for the distribution).

With slight changes in the transition statements, a new distribution can be generated which gives a distribution of the time between an event (e.g., a response) and another event (e.g., the end of a trial) in the following manner.

$$
\begin{aligned}
& 5.5,1, \\
& \text { si, } \\
& \text { S2, }
\end{aligned}
$$

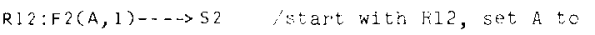

$$
\begin{aligned}
& l^{\prime \prime}: F 1(A, 1,10) \rightarrow-\rightarrow S 2 \text { /every second increment } \\
& R 1: F 2(A, 1) \cdots S 2 \text { / value of } A \\
& \text { levery resporise sets } A
\end{aligned}
$$

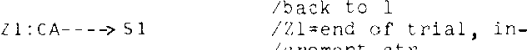

One of the more common measures employed in the behavioral sciences is that of the IRTs. This measure is taken easily with the aid of the F functions of SKED. For example:

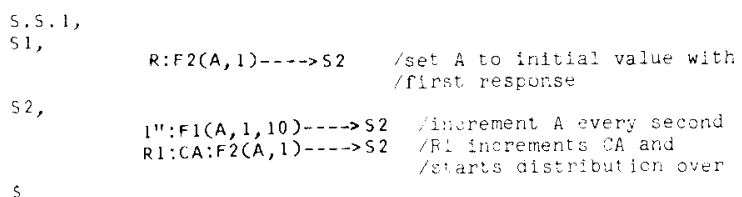

In a two-response (or more situation, all possible IRTs may be taken by assuming that each of the possible IRTs, based on the most recent response, is going to occur, but incrementing only the appropriately tagged counter when the next response occurs.

This section does not, of course, exhaust the possible types of data recording by $\mathrm{F}$ functions. These examples are merely some of the more frequently used. All of the sorting procedures mentioned above could be done using the standard SKED notation found in the first section of this paper. The $F$ functions make these types of recording more efficient in terms of computer-core locations used to store the program or, in simpler terms, make the routines shorter. However, some types of recording are either impossible with standard SKED notation or are excessively wasteful of core locations. These problems are best handled using the $\mathrm{F} 3$ function of SKED.

\section{SECTION 3: THE USE OF F3S IN DATA RECORDING}

One characteristic of the generality of a programming language is the ease with which the programmer can 
write and integrate his own assembly-language programs with the main body of his programming language. SKED allows easy access to assembly language via the F3 function [See Snapper \& Hamilton (1974) for greater detail]. Basically, the F3 function has the format F3(ONNNN, argl, arg2,...) where ONNNN is an octal (0) number (NNNN) representing the absolute core location of the user's subroutine and argl, etc., are numbers or tags which the programer wishes to be accessible to his subroutine. Thus, for example, if the programmer needed to set a counter to a particular value (say to indicate the dump number) the calling sequence might look like this: F3(ONNNN,A,4). By careful programming, F3s can be written which are usable by all stations and which simplify the state notation or collect data which could not otherwise be collected.

One way of using F3s to collect data would be in allowing the SKED Run Time system to punch user-coded paper tapes. Another data recording F3 is the binary sequential dependency F3. Briefly, this F3 allows the user to keep a record of the serial sequence of two events, for the occurrence of the last seven events. This F3 updates itself, in relation to the last seven events, every time it is entered, and records all permutations of the event for sequences of 2-7. The actual routine could be extended to cover sequences of 12 , but a sequence of 12 events requires 4096 separate counters, which is one entire field. Thus, there would be no room for state tables. One way to use this F3 might be for state tables. One way to use this $F 3$ might be:

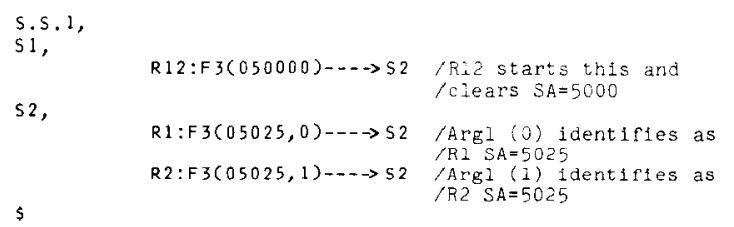

No reference to any recording counters is necessary in this F3. The binary sequential dependency F3 is actually two F3s which perform different functions. A variation of this recording $\mathrm{F} 3$ is the momentary maximizing $\mathrm{F} 3$. This F3 can record two events in a number of ways, but one way which will be illustrated is identical to that used by Shimp (1966) to study momentary maximizing. This program records the sequence of responses from the first response after reinforcement to the response that accumulates the next reinforcement. Unlike the binary sequential dependency $F 3$, this F3 records only one sequence (the final one) also up to seven in a sequence. This strategy actually requires three separate but interrelated F3s, allowing for a number of variations in use. The state table of the method described above would look like the following.

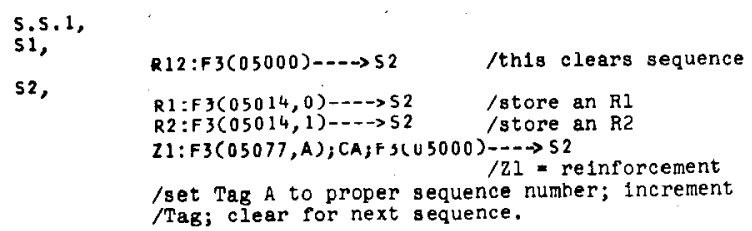

One last data-recording F3 will be presented. This F3 is the matrix F3. This F3 assesses the current value of one tagged distribution and resets another tag, which is itself a distribution, to a value corresponding to its place in the matrix. Consider the following problem. You wish to know the physical duration of a keypeck as a function of the IRT that peck concludes. If $A$ is a tag which is an IRT distribution and $B$ is a response-duration distribution, then this $\mathrm{F} 3$ would generate a $\mathrm{N}$ by $\mathrm{P}$ matrix with the rows being the response-duration distribution and the columns being the IRT length. The formula used to determine the proper counter number is $\mathrm{B}=(\mathrm{A} * \mathrm{~N})+\mathrm{B}$ where $\mathrm{N}$ is the number of counters involved in the $\mathrm{B}$ distribution. It could be used in the following manner:

$$
\begin{aligned}
& \text { S.S.1, } \\
& \$ 1, \\
& \text { s2, } \\
& \text { s3, } \\
& 54, \\
& \$ \\
& \begin{aligned}
R 12: F 2(A, 1) ; F 2(B, 1)--\rightarrow S 2 & \text { /both d1stributions } \\
& \text { /start on } 1
\end{aligned} \\
& \text { RI } \rightarrow \rightarrow S 3 \quad \text { /start IRTs } \\
& 1^{\prime \prime}: F 1(A, 1,10) \rightarrow \ldots S 3 \\
& R 1: C A \rightarrow-\rightarrow 54 \\
& 1^{\prime \prime}: F 1(A, 1,10)-->>54 \quad \text { /1ncrement duration } \\
& \text { /distribution } \\
& R 2: F 3(05000, B, A, 10) ; C B ; F 2(A, 1) ; F 2(B, 1) \cdots \rightarrow S 3 \\
& / \text { F3 sets matrix, record, }
\end{aligned}
$$

The F3s presented in this section are but a few of the possible F3s, but they serve to demonstrate the general strategies involved. By using a data-dumping F3 (DODUM), all of the data recorded by any strategy can be dumped onto a peripheral device for storage for off-line data analysis. If tags are used to increment counter numbers, based on time, then using the DODUM F3 can keep virtually every response as it occurred recorded on a peripheral device.

\section{SECTION 4: GUIDES TO DATA COLLECTION}

Each experimenter and each experiment require different types of data analysis. If this were not so, then one list of data-recording strategies would suffice. There are, however, a few guides for the programmer which can be stated.

(1) Determine which of the three generalized types of recording strategies best describes your situation. For instance, if your recording needs suggest a series of counters (that is, not just recording certain discrete events), each of which is related to the others, then you need either the F1 and F2 functions, or the F3 function. If the proposed recording is either an F1, F2, or an F3 function, then if the distribution is related by time, trials, or responses, you would probably use the F1 and F2 functions.

(2) If your recording strategy is feasible using any of the recording strategies, decide whether or not the programming can be integrated into the stimulus and contingency part of the state table. If it can be integrated, you will save space and the difficulty of 
keeping a parallel recording state set in synchrony with the state set of contingencies.

(3) If there is a choice between writing a new F3 and some other method, you should avoid writing your own F3 unless you are an experienced assembly language programmer.

F3s can be frustrating and may not be possible for anyone to write due to hardware or software considerations. For instance, a lot of time has been spent trying to insure that the OS/8 version of SKED can dump data on-line onto a DECTAPE. The fact of the matter is that when trying to dump more than seven complicated and independent stations, the DECTAPE is unable to interact with the Run Time system and store the data without occasional DECTAPE errors. This problem is solved by eliminating three ION commands and dumping data with the clock off.

(4) Regardless of the data strategy or mode of programming, debugging the program thoroughly is necessary. Program the experiment, load it into the Run Time system and then make known sequences of responses with known time values. Check the responses you have made against what the routine should have recorded. Errors in programming, and a general lack of understanding of what really was being recorded have gone undetected for long periods of time.

(5) The last general rule is a very basic one. Make very sure that you understand both the schedule and what it is you wished recorded. Many times, the person designing the experiment is not the person programming it. This brings us to the important point of communication. Communication is facilitated if you have some way to completely specifying your experiment so that others can understand your program without ambiguity. This, of course, brings us back to state notation.

These guides admittedly are not much help in specific situations. For those who are having trouble with a specific problem there is a means of possibility getting some help. This help might come from someone answering a request for help which you submit to the SKED Users Group for publication in the newsletter. Another SKED user may have already solved your problem and would be willing to help you with yours.

\section{REFERENCES}

Snapper, A., \& Hamilton, B. Programming special functions in the SKED system, Behavior Research Methods \& Instrumentations, 4, 1974, 173-176.

Snapper, A. G., \& Kadden, R. M. Time-sharing in a small computer based on a behavioral notation system. In $B$. Weiss (Ed.), Digital computers in the behavioral laboratory. New York: Appleton-Century-Crofts, 1970.

Shimp, C. P. Probabilistically reinforced choice behavior in pigeons. Journal of the Experimental Analysis of Behavior, 9 , $1966,443-455$. 